\title{
Signal Reconstruction from Nonuniformly Spaced Samples Using Evolutionary Slepian Transform-Based POCS
}

\author{
Jinsung Oh, ${ }^{1}$ Seda Senay, ${ }^{2}$ and Luis F. Chaparro (EURASIP Member) ${ }^{2}$ \\ ${ }^{1}$ Department of Electrical Engineering, Halla University, Wonju, Gangwon 220-712, Republic of Korea \\ ${ }^{2}$ Department of Electrical and Computer Engineering, University of Pittsburgh, 348 Benedum Hall, Pittsburgh, PA 15261, USA
}

Correspondence should be addressed to Luis F. Chaparro, chaparro@ee.pitt.edu

Received 29 January 2010; Accepted 15 June 2010

Academic Editor: Aydin Akan

Copyright ( 2010 Jinsung Oh et al. This is an open access article distributed under the Creative Commons Attribution License, which permits unrestricted use, distribution, and reproduction in any medium, provided the original work is properly cited.

\begin{abstract}
We consider the reconstruction of signals from nonuniformly spaced samples using a projection onto convex sets (POCSs) implemented with the evolutionary time-frequency transform. Signals of practical interest have finite time support and are nearly band-limited, and as such can be better represented by Slepian functions than by sinc functions. The evolutionary spectral theory provides a time-frequency representation of nonstationary signals, and for deterministic signals the kernel of the evolutionary representation can be derived from a Slepian projection of the signal. The representation of low pass and band pass signals is thus efficiently done by means of the Slepian functions. Assuming the given nonuniformly spaced samples are from a signal satisfying the finite time support and the essential band-limitedness conditions with a known center frequency, imposing time and frequency limitations in the evolutionary transformation permit us to reconstruct the signal iteratively. Restricting the signal to a known finite time and frequency support, a closed convex set, the projection generated by the time-frequency transformation converges into a close approximation to the original signal. Simulation results illustrate the evolutionary Slepian-based transform in the representation and reconstruction of signals from irregularly-spaced and contiguous lost samples.
\end{abstract}

\section{Introduction}

The problem of signal reconstruction from Nonuniformly spaced samples is central in many practical problems in image and signal processing [1-11]. Nonuniform sampling is a common result of Nyquist-Shannon sampling caused by jittering in the sampler, but it is also the case when samples are missing, either according to some distribution or in segments.

Reconstruction of Nonuniformly sampled signals can be approached numerically by considering sinc basis as frames [12]. Unfortunately, it is an ill-posed problem given the characteristics of the basis. Signals in practice have finite supports and can be approximated as nearly band-limited signals, based on this in $[13,14]$ it is shown that a more appropriate basis for signal interpolation is the Prolate Spheroidal Wave or Slepian functions [15]. It is thus possible to develop the projection of signals of finite time support that are nearly band-limited. The representation of bandpass signals, as the modulation of baseband components, can be obtained using modulated Slepian basis. The discrete prolate spheroidal sequences (DPSSs) are the discrete form of the prolate spheroidal wave functions (PSWFs) [16] and can be used as the basis for the projection of the sampled signal. Using DPSS as an orthogonal basis [14, 17], it is shown to reduce the sampling rate and reconstruction error.

The reconstruction of finite energy signals can thus be viewed as an interpolation or an estimation problem in which projection of the observed signal minimizes an error criteria. Constraining the solution to satisfy time and frequency conditions iteratively, a close approximation to the signal, with the given samples, is obtained. This is the basic idea of the projection onto convex sets (POCSs) method. This method was introduced by $[1,2]$ as an iterative algorithm for signal restoration. Since then, the POCS method has been successfully used in many signal and image recovery problems [3-11]. Time-frequency signal representations using short-time [9] and fractional Fourier transform $[10,11]$ have been recently used to implement this type of reconstruction. 
To obtain the POCS iterative solution, we consider that the signals of interest have a finite time support and an approximately finite frequency support. As such, the Slepian projection are used for this class of signals. To jointly consider time and frequency constraints, we develop a time-frequency representation from the Slepian projection. This can be done using the evolutionary spectral theory [18], where a signal can be represented in terms of a kernel which in turn can be obtained from the windowed signal. The magnitude square of the kernel is associated with the way the energy of the signal is distributed in time and frequency. It is also possible to obtain a similar representation, the discrete evolutionary transform (DET), for deterministic signals having components with time-varying frequency components [19]. Imposing time and frequency limitations in the DET permits us to reconstruct the signal iteratively, that is, the iterative projection generated by the time-frequency transformation converges into a close approximation to the original signal with the given Nonuniform samples.

The rest of the paper is organized as follows. In Section 2, we consider the reconstruction of Nonuniformly sampled signals. In this section we show why the PSWF basis is more appropriate than the sinc basis for the reconstruction from Nonuniform samples when the signal is of finite time support and essentially band-limited. In Section 3 we propose the time-frequency discrete evolutionary transform (DET) as the projection operator for the implementation of a recursive projection onto complex sets (POCSs) to recover missing samples. Assuming that the baseband components of a bandpass signal has finite support in time and frequency, a DET based in PSWF or Slepian functions is possible. This will be presented in Section 4. In Section 5 we illustrate the Slepian-based DET, and its application in the reconstruction of signals missing samples randomly and in blocks. Conclusions follow.

\section{Reconstruction of Nonuniformly Sampled Signals}

The sinc interpolation obtained in the Shannon-Nyquist sampling theory [20] for finite energy signals is fundamental in signal processing. However, it has some limitations. These limitations are (i) that the signal $x(t)$ is sampled uniformly at $n T_{s}$, for some sampling period $T_{s}$, and (ii) that $x(t)$ is band-limited, that is, $\Omega_{\max }$ is the maximum frequency present in the signal. Under these conditions the signal can be reconstructed from the uniform samples $\left\{x\left(n T_{s}\right)\right\}$ according to the following sinc interpolation:

$$
x(t)=\sum_{k} x\left(k T_{s}\right) S\left(t-k T_{s}\right),
$$

where $S(\cdot)$ is the sinc function. Several issues of practical interest arise when numerically implementing this interpolation. Besides the infinite dimension of the problem, uniform sampling is not realistic. Moreover, band-limitedness is just an approximation to reality.

If Nonuniform samples are available, the signal reconstruction suffers from dimensionality and ill-conditioning
[12]. Indeed, if the set of time-shifted sinc functions is considered a frame for $x(t)$, a Gramian matrix equation represents the following interpolation:

$$
\mathrm{Sc}=\mathbf{x}
$$

where $\mathbf{S}$ is a matrix containing shifted sinc functions, $\mathbf{x}$ is a vector with entries the samples of the signal, and $\mathbf{c}$ are the expansion coefficients of the projection in terms of the sinc functions. The infinite dimension of this problem makes it unsolvable, and when the dimension is reduced the problem becomes ill-posed [12].

The problem in part is due to using shifted sinc functions as basis: these functions are not appropriate for the interpolation given that time-limited signals are of infinite frequency support according to the uncertainty principle. A more appropriate basis is given by the Prolate Spheroidal Wave functions (PSWFs) [15].

The PSWFs are real-valued functions, with finite time support $2 T$, that maximize their energy in a given bandwidth. These functions $\left\{\psi_{n}(t)\right\}$ are the eigenfunctions of the sinc-based integral equation

$$
\psi_{n}(t)=\frac{1}{\lambda_{n}} \int_{-T}^{T} \psi_{n}(x) S(t-x) d x,
$$

where $\lambda_{n}$ is the eigenvalue corresponding to the eigenfunction $\psi_{n}(t)$. Given the orthogonality of the sinc functions, the above definition leads to the orthogonality of the PSWF functions, so that they, like the sinc functions, are basis for finite energy signals. Thus the sinc function $S(\cdot)$, which belongs to the space of band-limited signals, can be expanded in terms of the PSWFs as

$$
S\left(t-k T_{s}\right)=\sum_{m=0}^{\infty} \psi_{m}\left(k T_{s}\right) \psi_{m}(t) .
$$

Replacing this equation in the sinc interpolation gives an interpolation in terms of the PSWFs:

$$
x(t)=\sum_{m=0}^{\infty} \gamma_{m} \psi_{m}(t)
$$

with expansion coefficients

$$
\gamma_{m}=\sum_{k=0}^{\infty} x\left(k T_{s}\right) \psi_{m}\left(k T_{s}\right) .
$$

If the signal $x(t)$ has a finite support in time and approximately finite support in frequency, the above sums become finite. In that case, the upper limit of the sum in (5) depends on the approximately maximum frequency of $x(t)$, and in (6) the upper limit of the sum depends on the finite support of $x(t)$. A sampled-version of the signal could then be

$$
x\left(n T_{s}\right)=\sum_{k=0}^{N-1} \sum_{m=0}^{M-1} x\left(k T_{s}\right) \psi_{m}\left(n T_{s}\right) \psi_{m}\left(k T_{s}\right),
$$

where $N$ relates to the time support, and $M$ to the frequency support of $x(t)$. 
Thus, if $x(t)$ is time-limited and essentially band-limited, (5) and (6) provide a reconstruction of the signal from Nonuniformly sampled signals. In [14], it is shown that in the case of jittering sampling, that is, a subset of the uniform samples $\left\{x\left(n T_{s}\right)\right\}$ is available, the signal can be reconstructed.

A discrete version of the PSWF is used in these cases. The discrete PSW functions (or discrete PSW sequences or DPSS) are parameterized by the time bandwidth product $N W$ where $N$ is their length and $W=f T_{s}$ its normalized bandwidth. Like their analog counterparts, they are defined as the solution to the eigenvalue problem

$$
\lambda_{k} \psi_{k}(m)=\sum_{n=0}^{N-1} \frac{\sin (2 \pi W(n-m))}{\pi(n-m)} \psi_{k}(n)
$$

where $0 \leq n, m \leq N-1$, and $0 \leq W \leq 1 / 2$. The $N$ real eigenfunctions $\psi_{k}(n)$ are the DPSS, and the corresponding eigenvalues relate to the their energy concentration. The DPSS are also orthonormal.

\section{Evolutionary Spectral Representation and POCS}

The spectral representation of a stationary signal consists of a superposition of sinusoids, of all possible frequencies, with randomly varying amplitudes and phases. To obtain a similar representation for non-stationary signals, one can consider the Wold-Cramer representation [18] characterizing a nonstationary signal as the output of a linear time-varying system with a stationary white noise as input. Thus, a discrete non-stationary signal $x(n)$ can be expressed as

$$
x(n)=\sum_{k} X\left(n, \omega_{k}\right) e^{j \omega_{k} n},
$$

where $X\left(n, \omega_{k}\right)$ is an evolutionary kernel. The evolutionary spectrum of $x(n)$ is given by $\left|X\left(n, \omega_{k}\right)\right|^{2}$.

In [19], it is shown that the above representation can be extended to deterministic signals. The discrete evolutionary transform (DET) obtained in there is a generalization of the short-time Fourier transform as the evolutionary kernel $X\left(n, \omega_{k}\right)$ is obtained in term of the signal windowed, but the window in the DET varies with time and frequency. Thus the kernel is

$$
X\left(n, \omega_{k}\right)=\sum_{m=0}^{N-1} x(m) W_{k}(n, m) e^{-j \omega_{k} m}
$$

where $W_{k}(n, m)$ is the window which can be expressed in nonorthogonal functions such as Gabor's, or orthogonal functions such as Malvar's [19].

The POCS framework enables an iterative recovery algorithm incorporating time and frequency constraints. A desired signal $x(n)$ is assumed to lie in the region defined by the intersection of all the convex sets, that is,

$$
x(n) \in C_{0}, \quad C_{0}=\bigcap_{i} C_{i},
$$

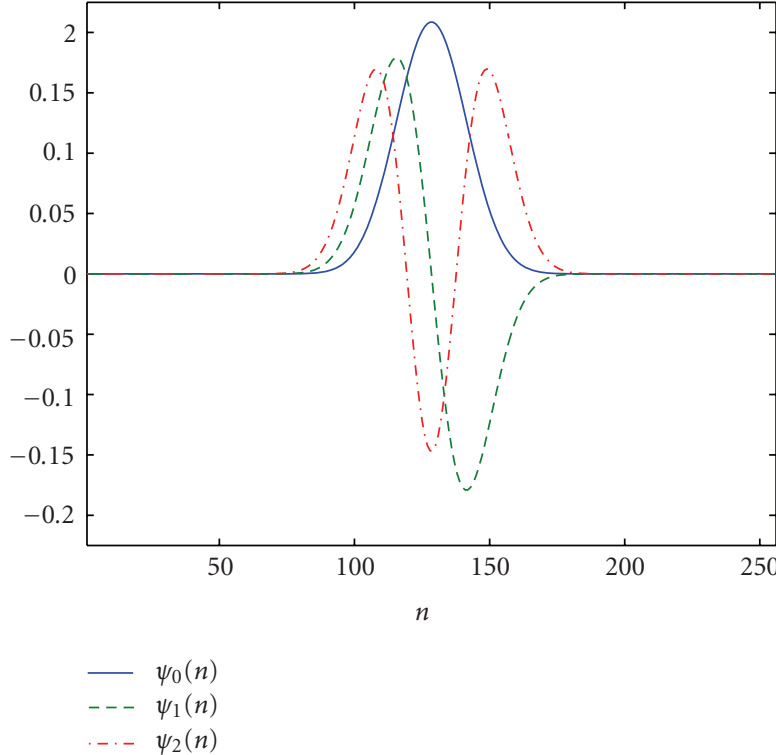

FIGURE 1: Baseband DPSS for $N=256, W=1 / 8$, and $K=64$ (note that $K=\lfloor 2 N W\rfloor)$.

where $C_{i}$ denotes the $i$ th closed convex set. Thus, the original signal can be restored by using the projection operators $P_{i}$ onto each convex set $C_{i}$. The general form of the POCS reconstruction is

$$
x_{(i+1)}(n)=P_{i}\left[x_{(i)}(n)\right],
$$

where $x_{(i)}(n)$ is the reconstructed signal after $i$ iterations. Assuming that the signal of interest is square summable and that the DET projects a signal into another square summable signal, under joint time-frequency constraints we develop an iterative POCS algorithm to recover the signal from partial information of it.

\section{Evolutionary Slepian Transform-Based POCS}

A bandpass real-valued signal $x(t)$ can be also represented in terms of baseband signals as

$$
x(t)=\operatorname{Re}\left[(a(t)+j b(t)) e^{-j \Omega_{0} t}\right],
$$

where $a(t)$ and $b(t)$ are low pass signals and $\Omega_{0}$ is the center frequency of the Fourier transform of $x(t)$ and $X(\Omega)$. Assuming the $a(t)$ and $b(t)$ components have finite time support and are essentially band-limited, we can represent them using the Slepian projection presented above. In that case, the signal $x(t)$ can be expressed as

$$
x(t)=\operatorname{Re}\left[\sum_{m=0}^{\infty}\left(\gamma_{m}+j \eta_{m}\right) \psi_{m}(t) e^{-j \Omega_{0} t}\right],
$$

that is, in terms of modulated Slepian functions.

The bandpass DPSS $[21,22]$ which have the highest energy concentration in a given passband are defined by

$$
\xi_{k}(n)=e^{-j 2 \pi W_{c} n} \psi_{k}(n),
$$




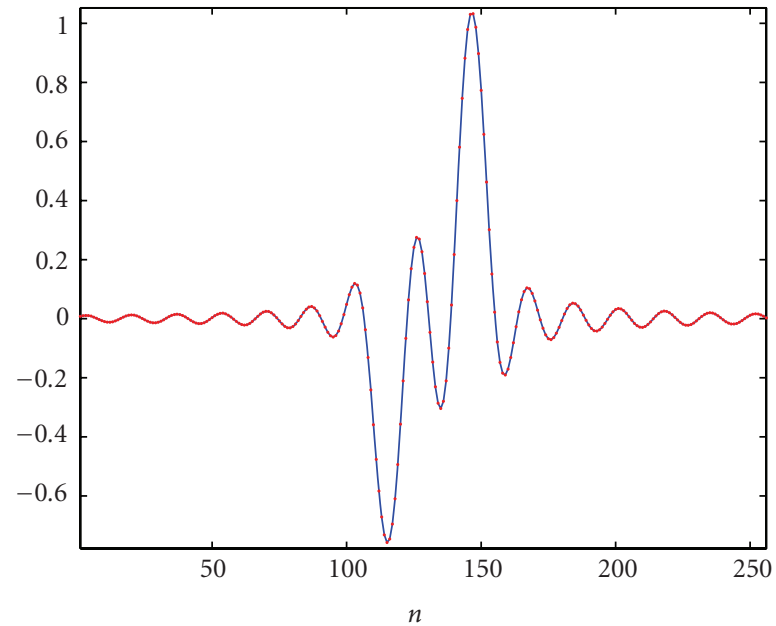

(a)

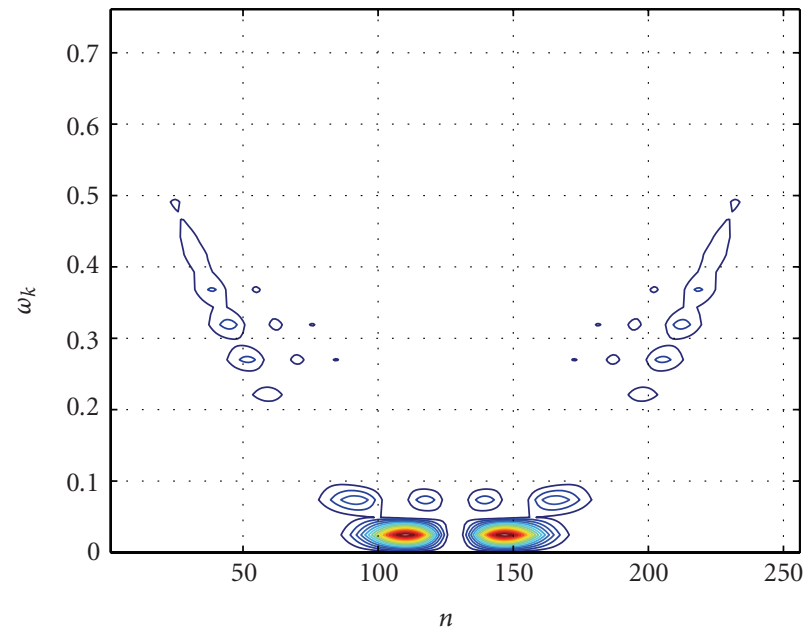

(b)

FIGURE 2: An example of evolutionary Slepian spectrum: (a) $x_{1}(n),(\mathrm{b})\left|X\left(n, \omega_{k}\right)\right|^{2}$.

where the passband is $\left[0 \leq W_{c}-W, W_{c}+W \leq 1 / 2\right]$. When the signal energy outside the given frequency band is very small,

$$
\frac{1}{2 \pi} \int_{\omega \notin\left[W_{c}-W, W_{c}+W\right]}|X(\omega)|^{2} d \omega \approx 0,
$$

the bandpass DPSS provide an efficient representation of passband signals and accurate channel estimation [21, 22]. Allowing the center frequency to vary from zero to infinity (or zero to $\pi$ in the discrete domain) we then have a general representation for any signal.

The general representation for a complex signal $x(t)$ in terms of the PSWFs is given by

$$
x(t)=\sum_{m=0}^{\infty} \gamma_{m} \xi_{m}(t), \quad \gamma_{m}=\sum_{k=0}^{\infty} x\left(k T_{s}\right) \xi_{m}\left(k T_{s}\right),
$$

where $\left\{\xi_{m}(t)\right\}$ can be the band-pass or the base band (when the center frequency is zero) Slepian functions. If the signal $x(t)$ is time limited, and essentially in the frequency bands $\Omega_{c} \pm \Omega$ and $-\Omega_{c} \pm \Omega$, then (7) becomes

$$
x(t)=\sum_{m=0}^{M-1} \sum_{m=0}^{N-1} x\left(k T_{s}\right) \xi_{m}\left(k T_{s}\right) \xi_{m}(t)
$$

where, as indicated before, $M$ depends on the frequency support and $N$ on the time support.

In [14], the reconstruction of the original signal from a given set of Nonuniform samples is considered, while the effect of the distribution of the Nonuniform samples in the reconstruction is studied in [23]. Assuming that $q$ samples $x\left(k_{i} T_{s}\right), i=0,1, \ldots, q-1$ are missing, then letting $\mathbf{u}$ be the $q$-dimensional vector of unknown samples and $t=k_{i} T_{s}$ we obtain from above

$$
\mathbf{u}=\Lambda \mathbf{u}+\mathbf{g}
$$

where $\Lambda$ is a matrix with a subset of the entries of the matrix generated by the terms in square brackets in (18) and

$$
g(t)=\sum_{k \notin \mathbf{u}} x\left(k T_{s}\right)\left[\sum_{m=0}^{M-1} \xi_{m}\left(k_{i} T_{s}\right) \xi_{m}(t)\right] .
$$

The missing samples are recovered if the above equation can be solved for $\mathbf{u}$, or if $\mathbf{I}-\boldsymbol{\Lambda}$ is invertible. Given the many possible ways the missing samples could be distributed, this might not be possible. However, as indicated in [12, 23] there are cases where reconstruction is possible with the sinc interpolation, and we will show later that it is also the case when we are using the Slepian POCS.

To apply joint time and frequency constraint in the POCS we develop a DET based on the Slepian representation of the signal. Suppose a discrete signal $x(n)$ can be represented in terms of some orthogonal basis $\left\{\phi_{k}(n)\right\}$,

$$
\begin{aligned}
x(n) & =\sum_{k=0}^{K-1} d_{k} \phi_{k}(n), \quad 0 \leq n \leq N-1, \\
d_{k} & =\sum_{n=0}^{N-1} x(n) \phi_{k}^{*}(n), \quad 0 \leq k \leq K-1,
\end{aligned}
$$

where $\left\{d_{k}\right\}$ are the expansion coefficients. Rewriting $x(n)$ as

$$
x(n)=\sum_{k=0}^{K-1} \underbrace{\left[d_{k} \phi_{k}(n) e^{-j \omega_{k} n}\right]}_{=X\left(n, \omega_{k}\right)} e^{j \omega_{k} n},
$$

where $\omega_{k}=2 \pi(k / N)$. The evolutionary kernel $X\left(n, \omega_{k}\right)$ can be expressed in terms of $x(n)$ by replacing the $d_{k}$ coefficients,

$$
\begin{aligned}
X\left(n, \omega_{k}\right) & =d_{k} \phi_{k}(n) e^{-j \omega_{k} n} \\
& =\sum_{m=0}^{N-1} x(m) \underbrace{\left[\phi_{k}(n) \phi_{k}^{*}(m) e^{-j \omega_{k}(n-m)}\right]}_{=W_{k}(n, m)} e^{-j \omega_{k} m} .
\end{aligned}
$$



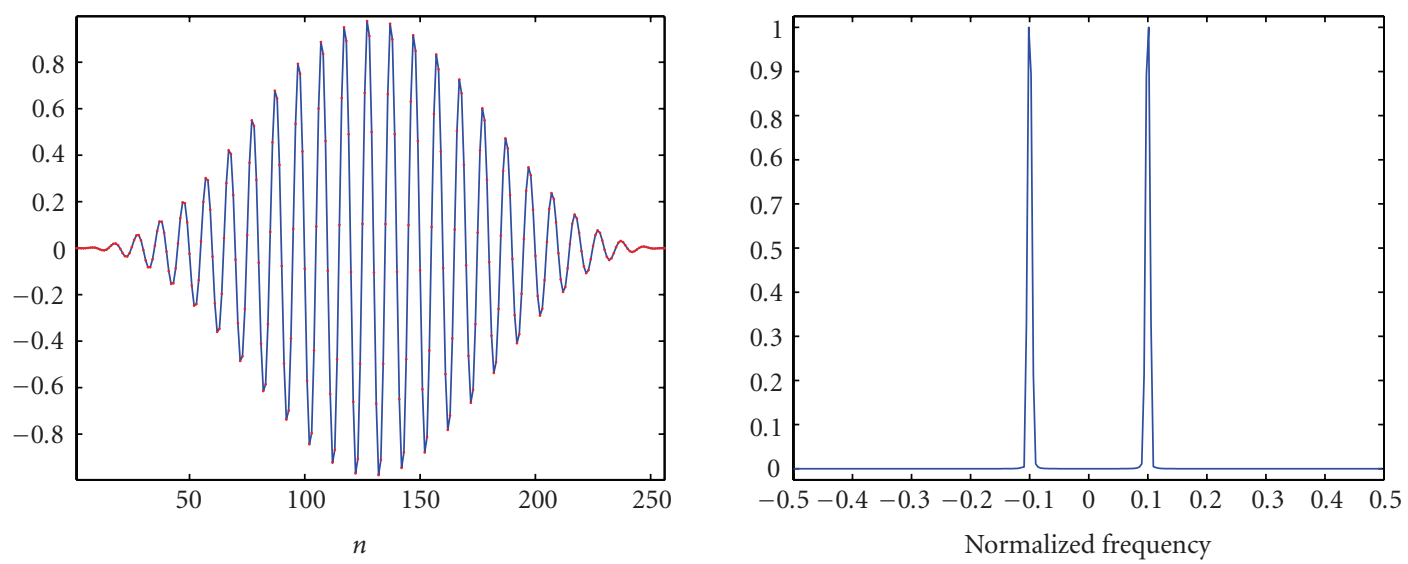

(a)
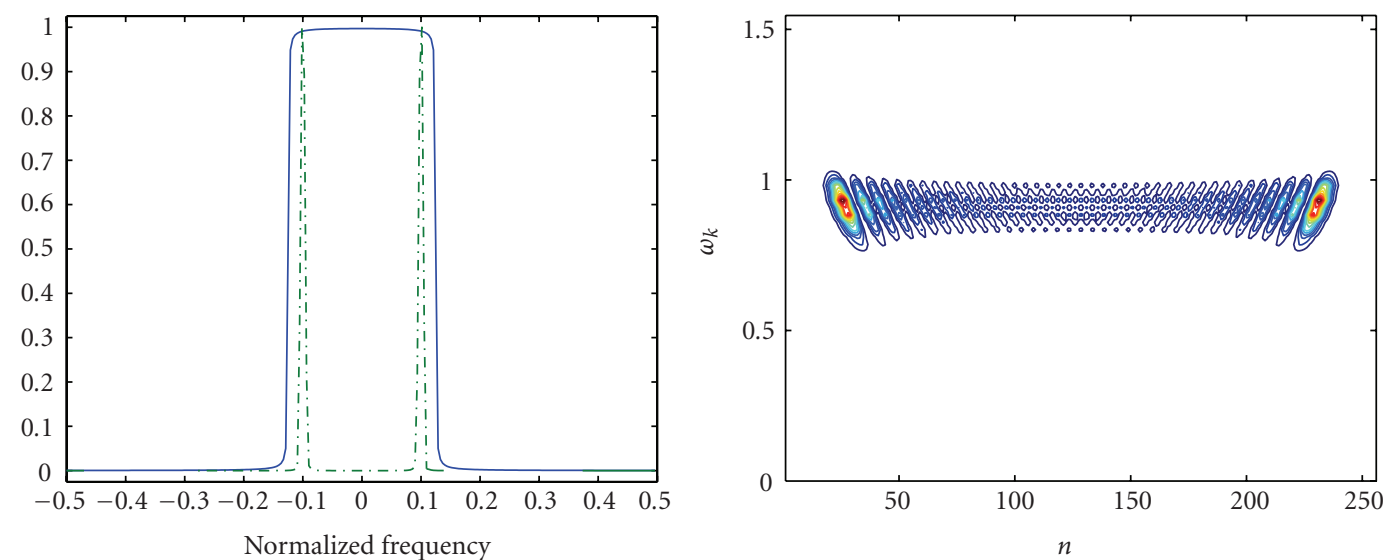

$-\Sigma_{k=0}^{K-1}\left|\psi_{k}(\omega)\right|$

... $|X(\omega)|$

(b)
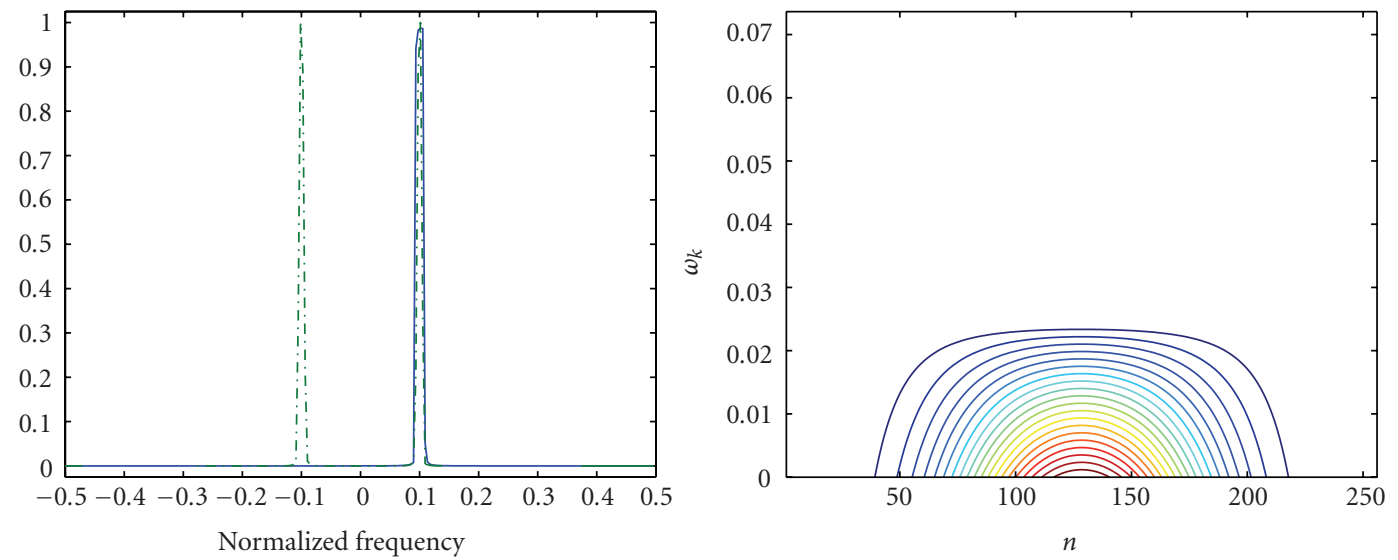

$-\sum_{k=0}^{K-1}\left|\xi_{k}(\omega)\right|$

-. $|X(\omega)|$

(c)

Figure 3: Comparison of evolutionary Slepian spectrum for passband test signal using baseband and bandpass DPSS. (a) $x_{2}(n)$ and its spectrum $|X(\omega)|$, (b) spectrum of baseband DPSS $(K=64)$ and corresponding $\left|X\left(n, \omega_{k}\right)\right|^{2}$, and (c) spectrum of bandpass DPSS $(K=4)$ and corresponding $\left|G\left(n, \omega_{k}\right)\right|^{2}$. 


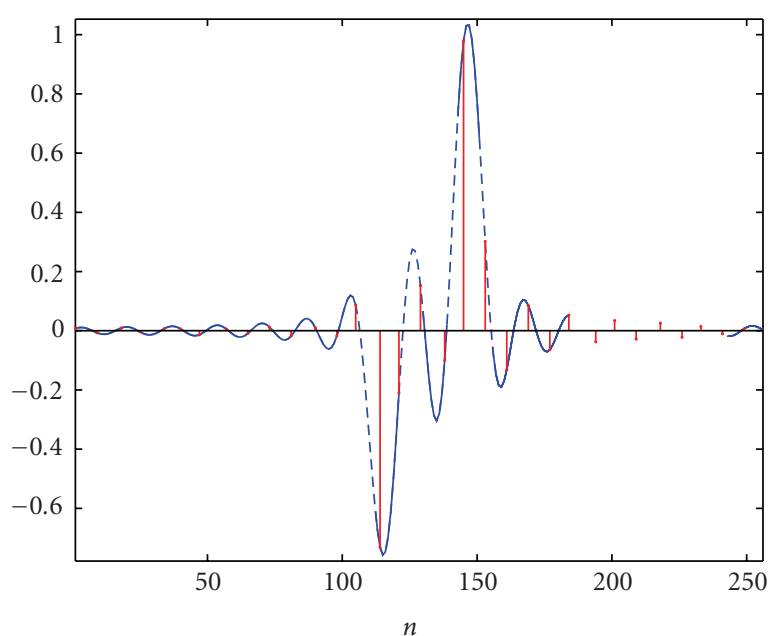

(a)

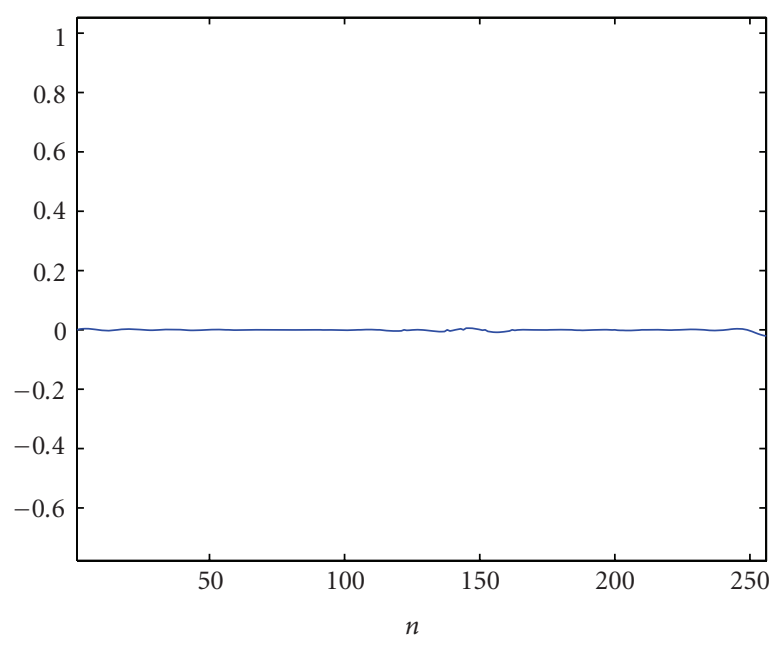

(c)

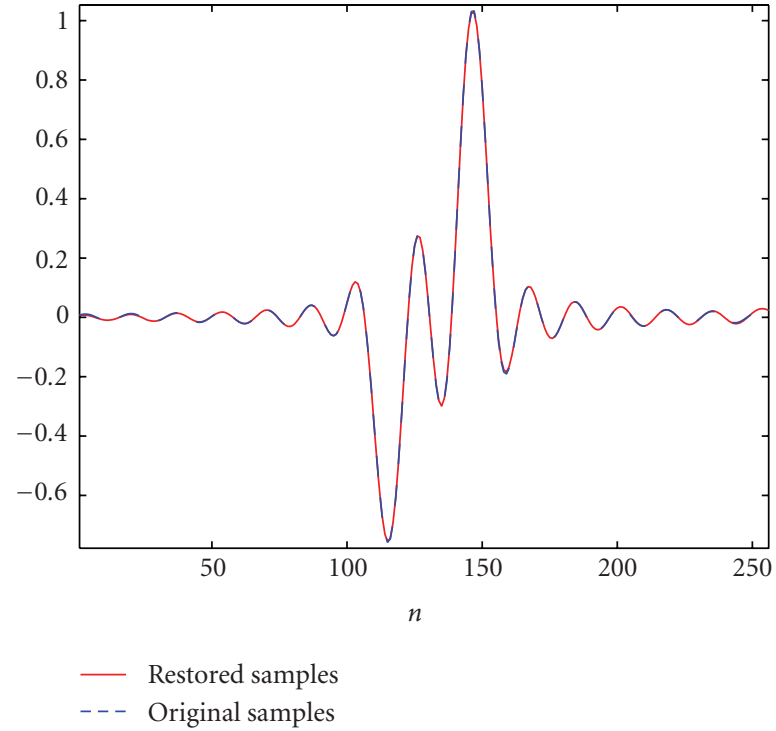

(b)

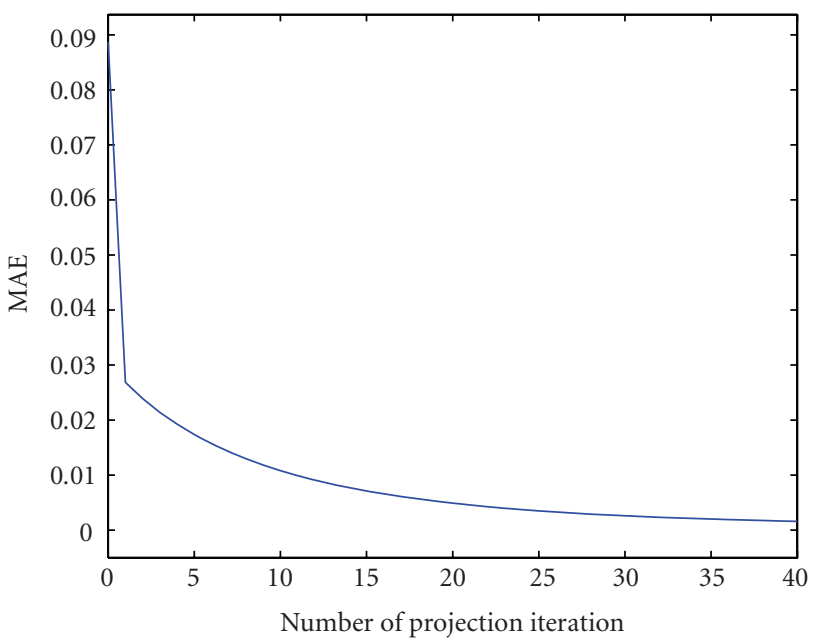

(d)

Figure 4: Restored results for the test signal $x_{1}(n)$ (a) Irregularly spaced subsamples $(L=8, \tau \sim \mathcal{N}(0,1))$, (b) restored signal, (c) error, and (d) convergence behaviour.

To obtain the evolutionary kernel, in particular the window $W_{k}(n, m)$, we consider the bandpass DPSS $\left\{\xi_{k}(n)\right\}$ as basis for the representation of baseband and passband signals. The window is then expressed as

$$
W_{k}(n, m)=\xi_{k}(n) \xi_{k}^{*}(m) e^{-j \omega_{k}(n-m)}
$$

It is important to understand that when the signal under consideration is modulated, that is, $x(n)=g(n) e^{-j \pi W_{c} n}$, and we use the bandpass Slepian functions, we can obtain the spectrum of $g(n)$ or $\left|G\left(n, \omega_{k}\right)\right|^{2}$. For a signal with bandpass characteristics, the signal can be represented by a small number of bandpass DPSS coefficients and then restored by small number of projection iteration compared to baseband DPSS based DET, which will be shown in next section.

\section{Simulation}

5.1. Slepian-Based DET. To illustrate the baseband and bandpass Slepian representation and theirSlepian-based DET, consider the test signals

Baseband signal: $\quad x_{1}(t)=\operatorname{sinc}(t-2.1)-0.7 \operatorname{sinc}(t+1.7)$,

$$
-15 \leq t \leq 15,
$$

Passband signal: $x_{2}(t)=\operatorname{sinc}^{2}\left(f_{B} t\right) \cos \left(2 \pi f_{C} t+\frac{\pi}{3}\right)$,

$$
-0.5 \leq t \leq 0.5,
$$

where $f_{C}=25.6 \mathrm{~Hz}$ is a carrier frequency, and $f_{B}=2 \mathrm{~Hz}$. Discrete signals $x_{1}(n)$ and $x_{2}(n), 0 \leq n \leq N-1, N=256$, are 


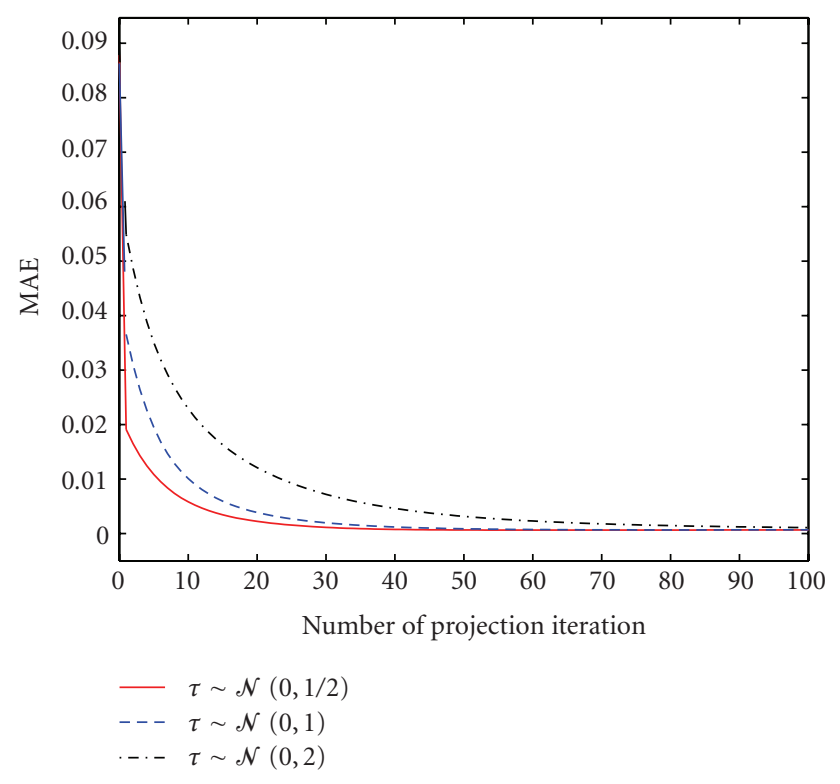

FIGURE 5: Convergence speed and MAE performance according to the degree of irregularity.

obtained from the uniform sampling on the signal $x_{1}(t)$ and $x_{2}(t)$, respectively, using a sampling period $T_{s}=30 / 256$ and $T_{s}=1 / 256 \mathrm{sec}$. For the signal $x_{1}(n)$, the evolutionary Slepian spectrum $\left|X\left(n, \omega_{k}\right)\right|^{2}$ using the baseband DPSS, shown in Figure 1, is illustrated in Figure 2. The evolutionary Slepian spectrum shows that the energy of the test signal $x_{1}(n)$ is highly concentrated in $n \in[100,160]$ and $\omega_{k} \in[0,1 / 4]$ rad. As shown in Figure 2(b), the DET provides accurate representation for a nearly time-limited and band-limited signal in the time-frequency domain.

The 256-point bandpass test signal $x_{2}(n)$ in Figure $3(\mathrm{a})$ is to be represented by the evolutionary Slepian spectrum. Its Fourier transform is shown in Figure 3(a) where the signal energy is concentrated at normalized frequency 0.1. In many practical applications, the exact frequency band of the signal is known. Therefore, if we have enough knowledge about the spectrum characteristics of the signal, we can represent the signal with small number of DPSS. Figure 3(b) shows the evolutionary Slepian spectrum for $x_{2}(n)$ using baseband DPSS with $K=64$ coefficients and normalized bandwidth of $W=1 / 8$. The evolutionary Slepian spectrum using bandpass DPSS is also shown in Figure 3(c) where $K=4, W=1 / 128$, and $W_{c}=0.1$. Therefore, if we project the signal that has bandpass characteristics on the DET domain, the signal can be restored only by a small number of basis, that is, bandpass DPSS with the same accuracy obtained from baseband DPSS.

5.2. Reconstruction of Irregularly Sampled Signals. In this section, we perform three different simulations to illustrate the effectiveness of DET-based POCS. We use the POCS methodology for reconstruction of nonuniformly sampled and band-limited signals.
5.2.1. Nonuniform Jittering Sampling with Known Distribution. Irregularly spaced samples $\left\{x\left(n_{i}\right)\right\}$ are obtained from the original signal $x(n)$ by $x\left(n_{i}\right)=x([n(N / L)+\tau])$ where $\tau$ is the timing jitter with normal distribution $\mathcal{N}\left(0, \sigma^{2}\right)$ and \lceil\rfloor denotes the nearest integer, and $L$ is a decimation factor.

Figure 4 (a) shows the irregularly spaced samples with 32 point $(L=8)$ from the test signal $x_{1}(n)$. In this simulation, the parameters for the baseband DPSS are $W=1 / 16$, and $K=32$. As shown in Figures 4(b), 4(c), and 4(d), the original signal can be recovered with very small error after 40 iterations. Figure 5 shows the relationship between the degree of irregularity $\left(\tau \sim \mathcal{N}\left(0, \sigma^{2}\right)\right)$ and the performance in terms of mean absolute error (MAE) and the speed of convergence. It is clear that the performance depends on the degree of irregularity. Figure 5 also suggests that, although the number of iterations should be increased according to the degree of irregularity, a nearly perfect reconstruction can be possible.

In case of nonstationary signals such as a chirp, the restored signal using bandpass DPSS-based DET is shown in Figure 6 . The results clearly indicate that the reconstruction of the Nonuniformly sampled nonstationary signal can be possible with very small error.

For a speech signal, the restored results using baseband and bandpass DPSS-based DET are shown in Figure 7. Its frequency components are also shown in Figure 7(b) showing that the energy of the signal is concentrated in a normalized frequency band $0.02<\omega<0.1$. Note that the bandpass DPSS-based DET projection converges faster than the baseband approach for a bandpass type signal. It is clear that the bandpass type signal can be restored only by a small number of components and the iteration, with the same MAE performance compared to baseband DPSS-based DET, converges faster. Therefore, the DET-based POCS algorithm provides a fast and accurate technique for recovering band-limited samples from the irregularly spaced subsamples.

5.2.2. Nonuniform Jittering Sampling with Unknown Distribution. In this simulation, we consider the extreme case of irregularly spaced, that is, randomly spaced subsamples. Figure 8 shows examples of restored signal from the randomly spaced subsamples for test signal $x_{1}(n)$ and the speech signal, given above. As shown in Figure 8, for a time-limited signal such as $x_{1}(n)$, the restored performance strongly depends on the sampling distribution. For the speech signal, since its energy is well distributed in the time domain, the restored signal is not sensitive to the distribution of sampling points. Note that the MAE values of the restored speech signals under 5 different random sampling patterns lie between 0.2 and 0.3 after convergence. This result clearly indicates that for a signal with uniformly distributed energy in the time domain, the DET based POCS algorithm is capable of signal recovery from randomly spaced subsamples. As pointed out in [23], if the gaps between missing samples due to randomly spaced are large, iterative technique such as POCS is more efficient than noniterative method. 


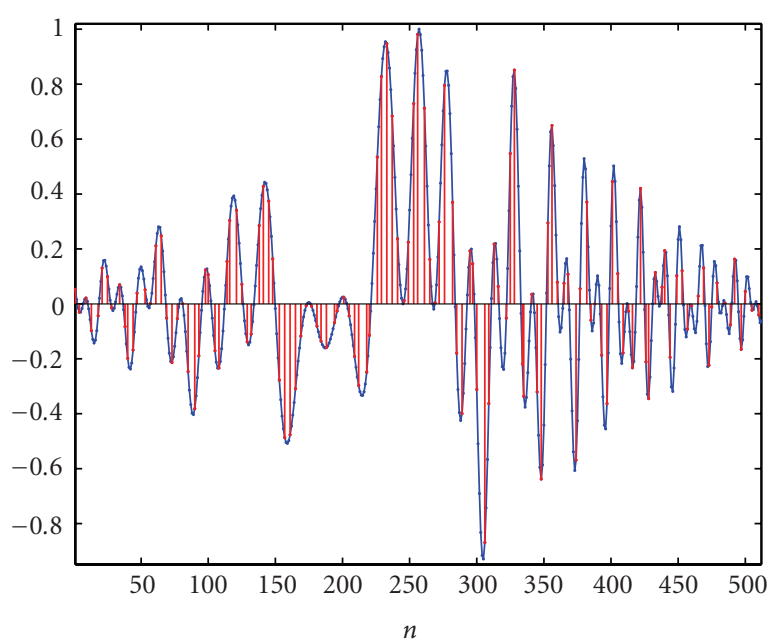

(a)

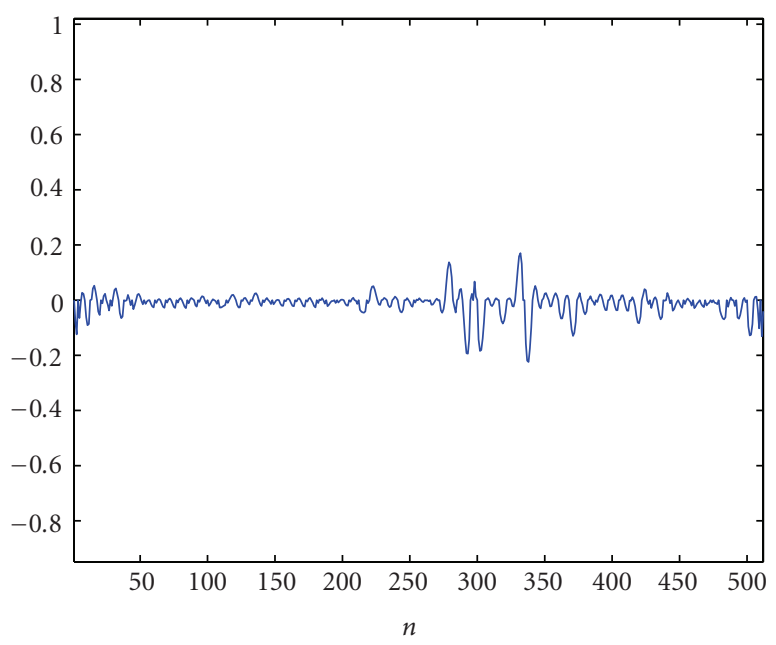

(c)

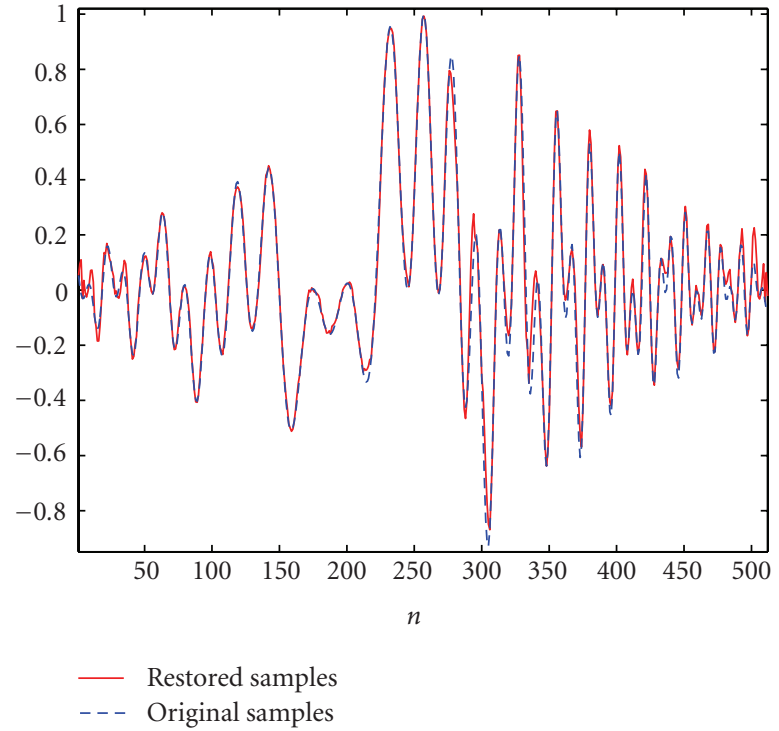

(b)

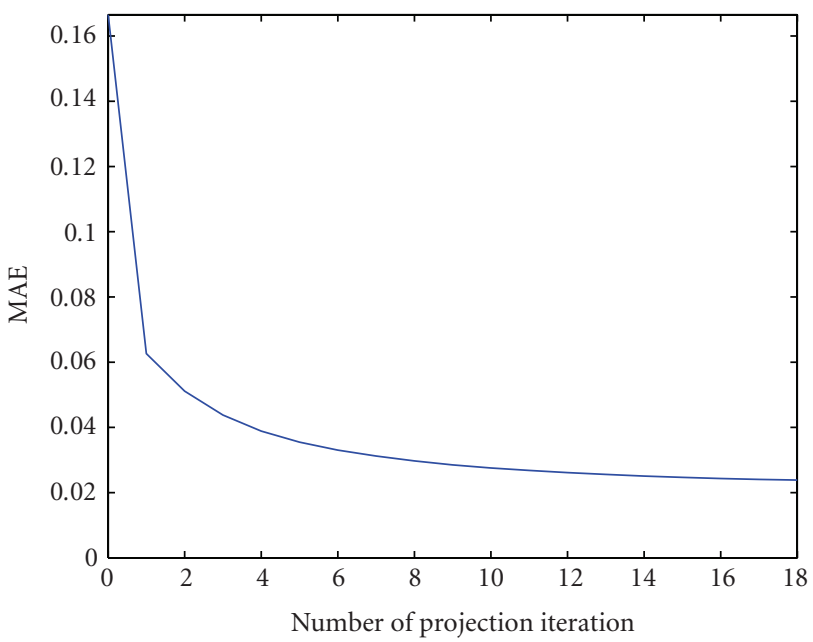

(d)

Figure 6: Restored results for the chirp signal $(N=512)$. (a) Irregularly-spaced subsamples $(L=4, \tau \sim \mathcal{N}(0,1 / 2))$, (b) restored signal by bandpass DPSS ( $K=64, W_{c}=1 / 16$ ) based DET, (c) error, (d) convergence behaviour.

5.2.3. Block or Contiguous Sample Losses. For the recovery of uniformly sampled signals with contiguous lost samples, the same projection methodology is applied in this simulation. A general assumption is that the lost data on the sampled signals compared to the total number of samples is small and that the available samples are representatives of the original signal [24]. Figure 9 shows examples of restored speech signal by bandpass DPSS- $(K=16)$ based DET from the uniformly spaced speech signal with continuous missing data. For larger values of missing samples, since the assumption does not hold due to severe loss of information on the signal, the performance of signal recovery is degraded. However, the proposed method shows very promising results as shown in Figure 9.

\section{Conclusions}

In this paper, we have introduced a new discrete evolutionary Slepian transform capable of efficient representation of band-limited signals. For the evolutionary kernel window, baseband and bandpass DPSS are used for the representation of baseband and bandpass signals, respectively. The evolutionary Slepian spectrum provides an accurate representation of time-and-band limited signal in the timefrequency domain. For the reconstruction, the DET-based POCS algorithm is applied in the area of signal recovery from nonuniformly spaced subsamples. For a signal that has bandpass characteristics, the signal can be represented by a small number of bandpass DPSS coefficients with the same 

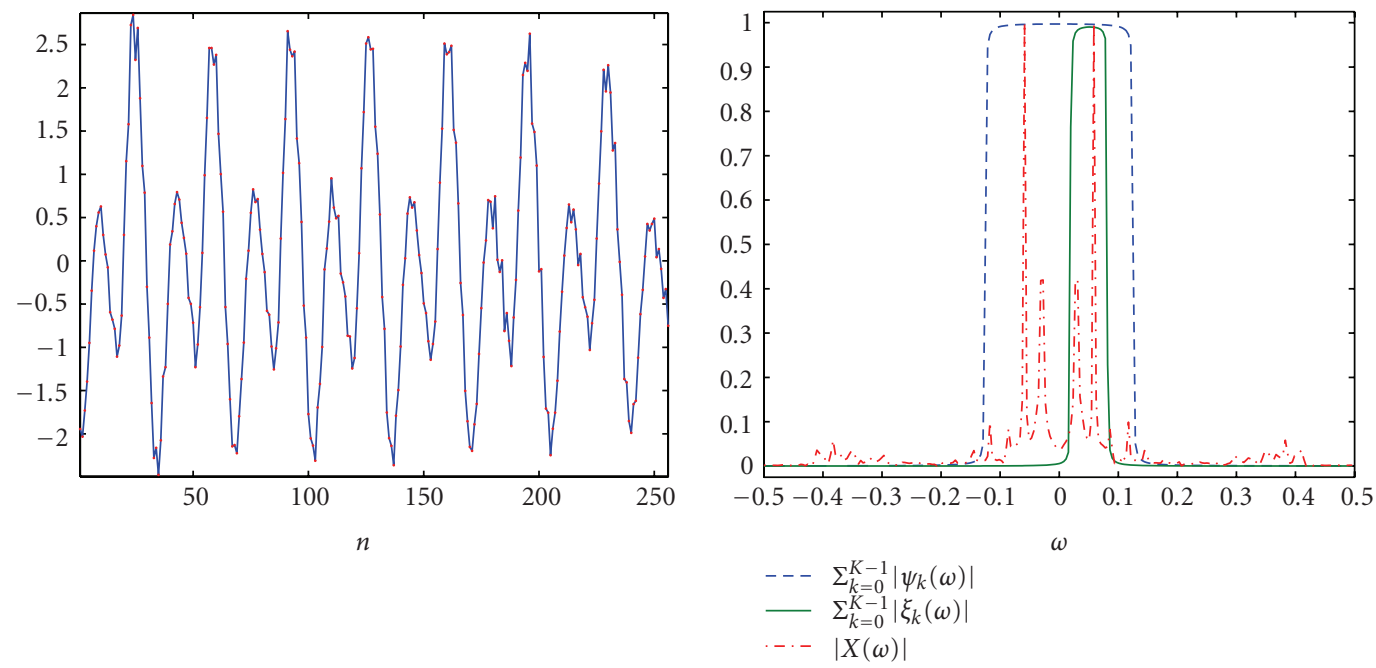

(a)
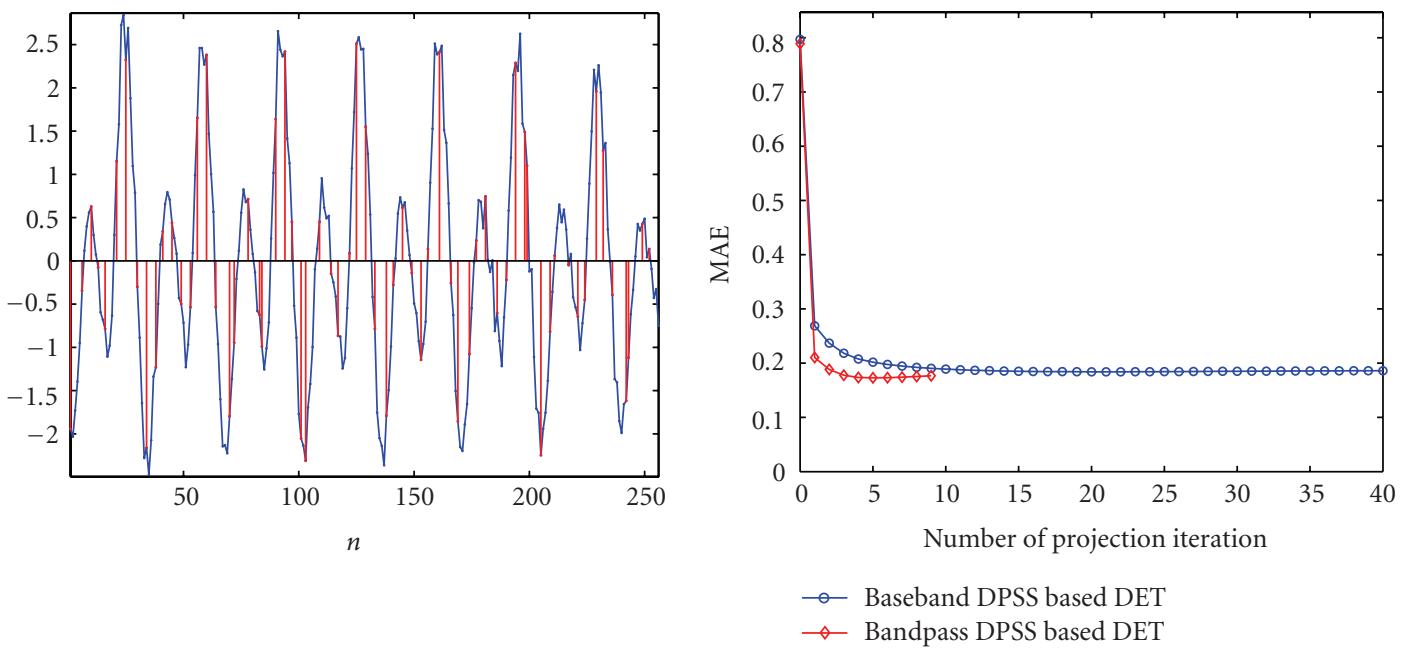

(c)

(d)
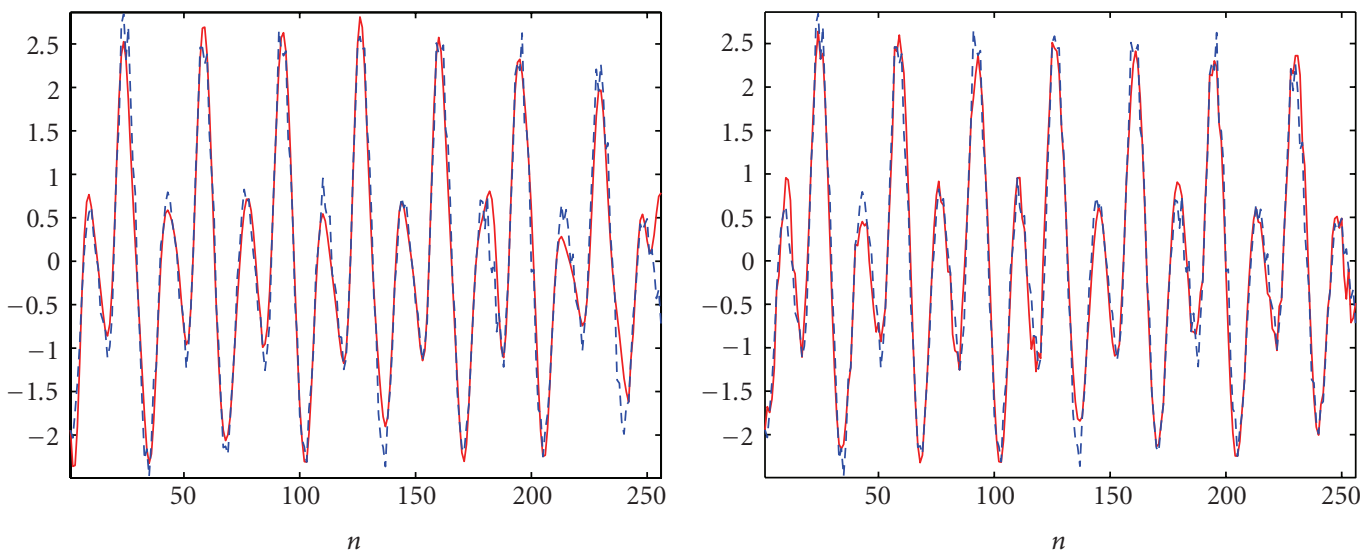

$\begin{array}{cc}- & \text { Restored samples } \\ --- & \text { Original samples }\end{array}$

$\begin{array}{ll}\text { - } & \text { Restored samples } \\ -- & \text { Original samples }\end{array}$

(f)

FIGURE 7: Restored results for the speech signal. (a) Speech signal, (b) spectrum of speech, baseband, and bandpass DPSS, (c) irregularly spaced subsamples $(L=4, \tau \sim \mathcal{N}(0,1 / 2))$, (d) convergence behaviours, (e) restored signal by baseband DPSS- $(K=64)$ based DET, and (f) restored signal by bandpass DPSS- $\left(K=16, W_{c}=0.05\right)$ based DET. 

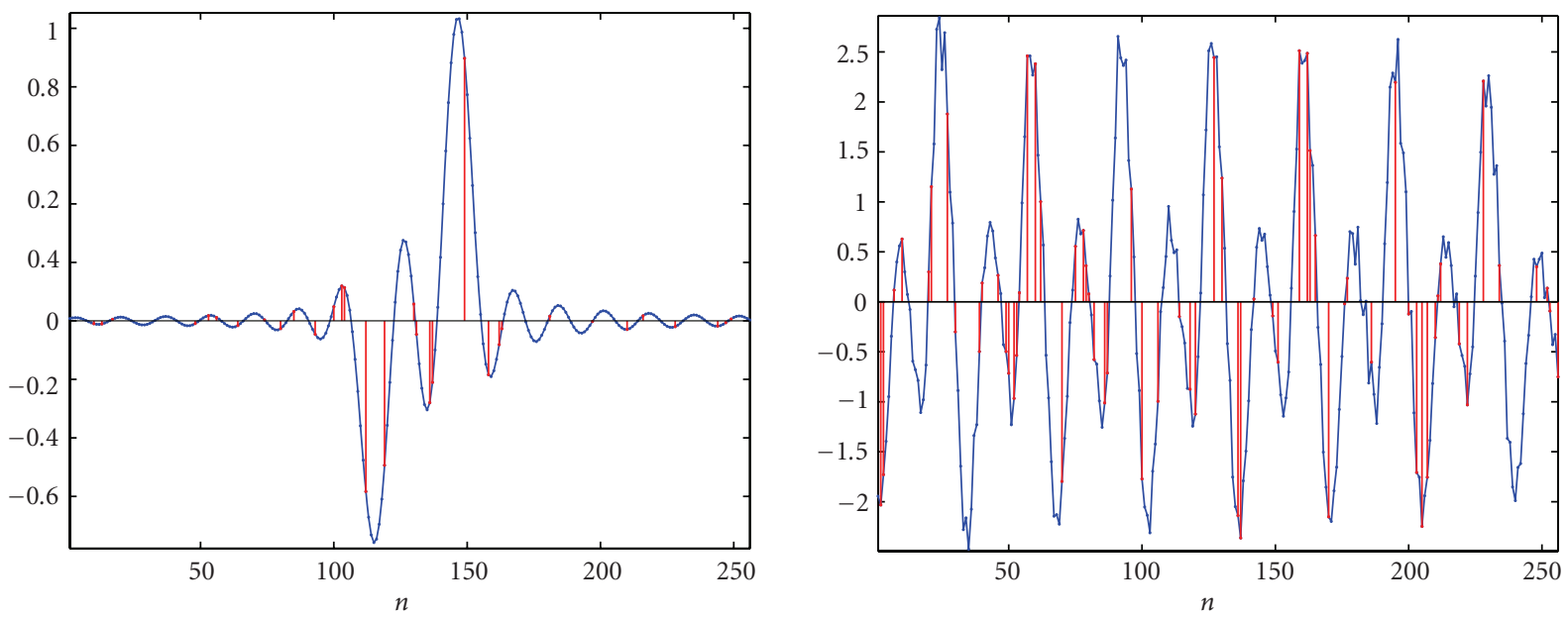

(a)
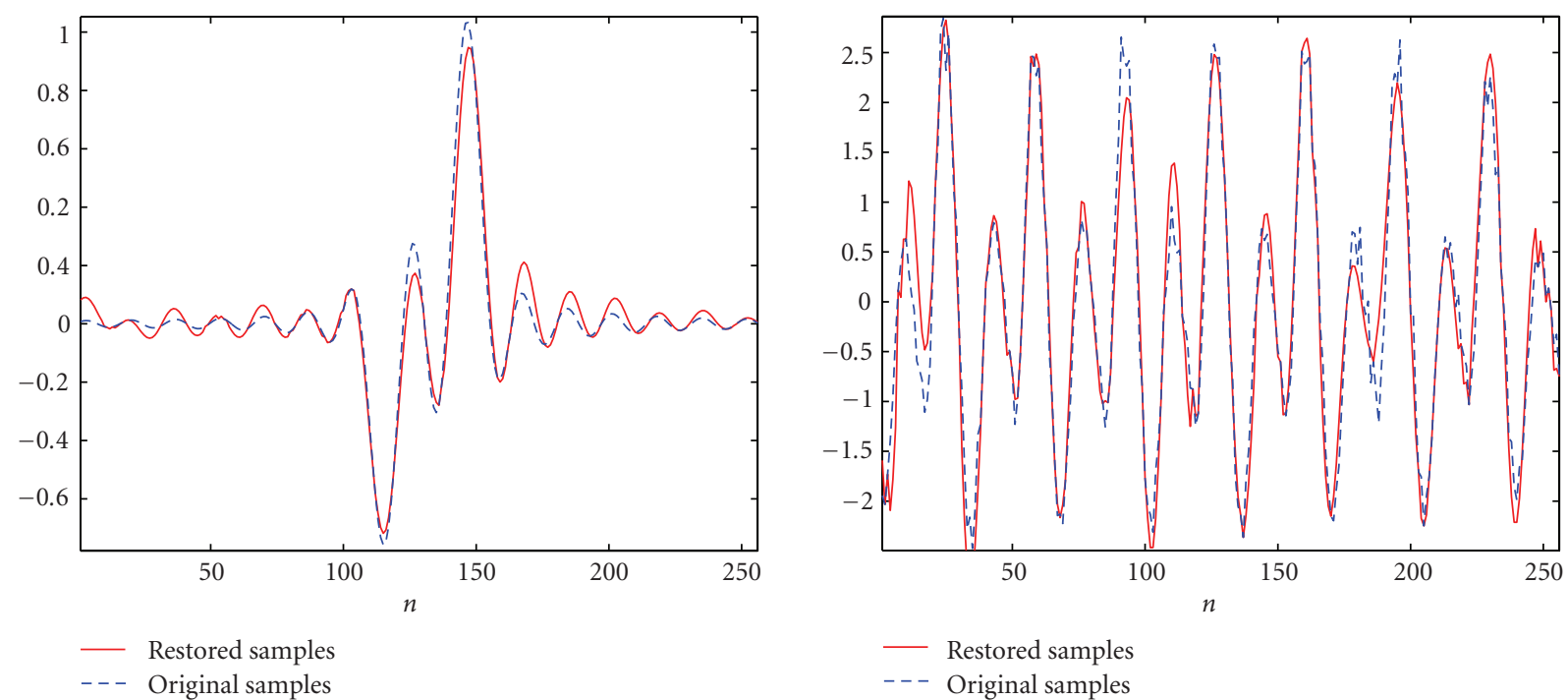

(b)
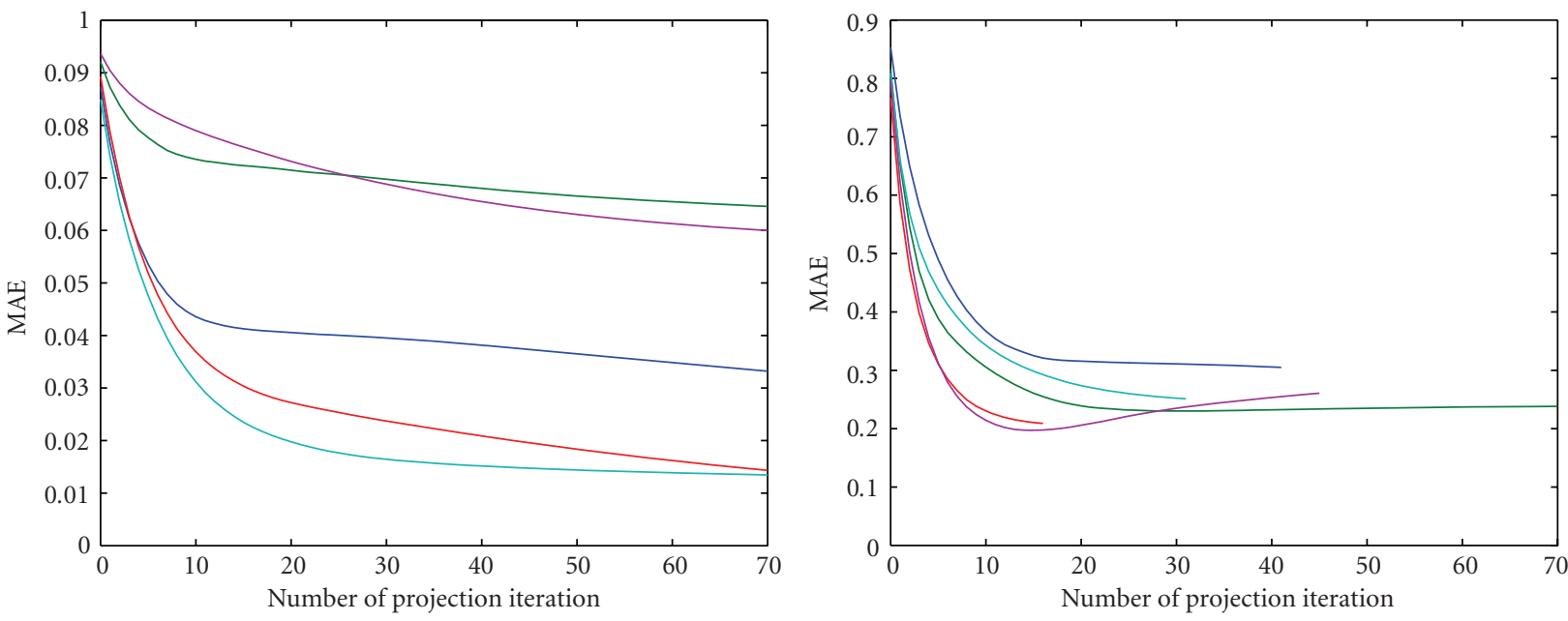

(c)

FIGURE 8: Restored results for randomly-spaced subsamples. (a) Randomly spaced subsampled test and speech signals, (b) restored test signal by baseband DPSS-based DET $(L=8, K=32)$ and restored speech signal by bandpass DPSS-based DET $(L=4, K=16)$, and $(\mathrm{c})$ convergence behaviours of 5 different random sampling patterns (left: test signal, right: speech signal). 

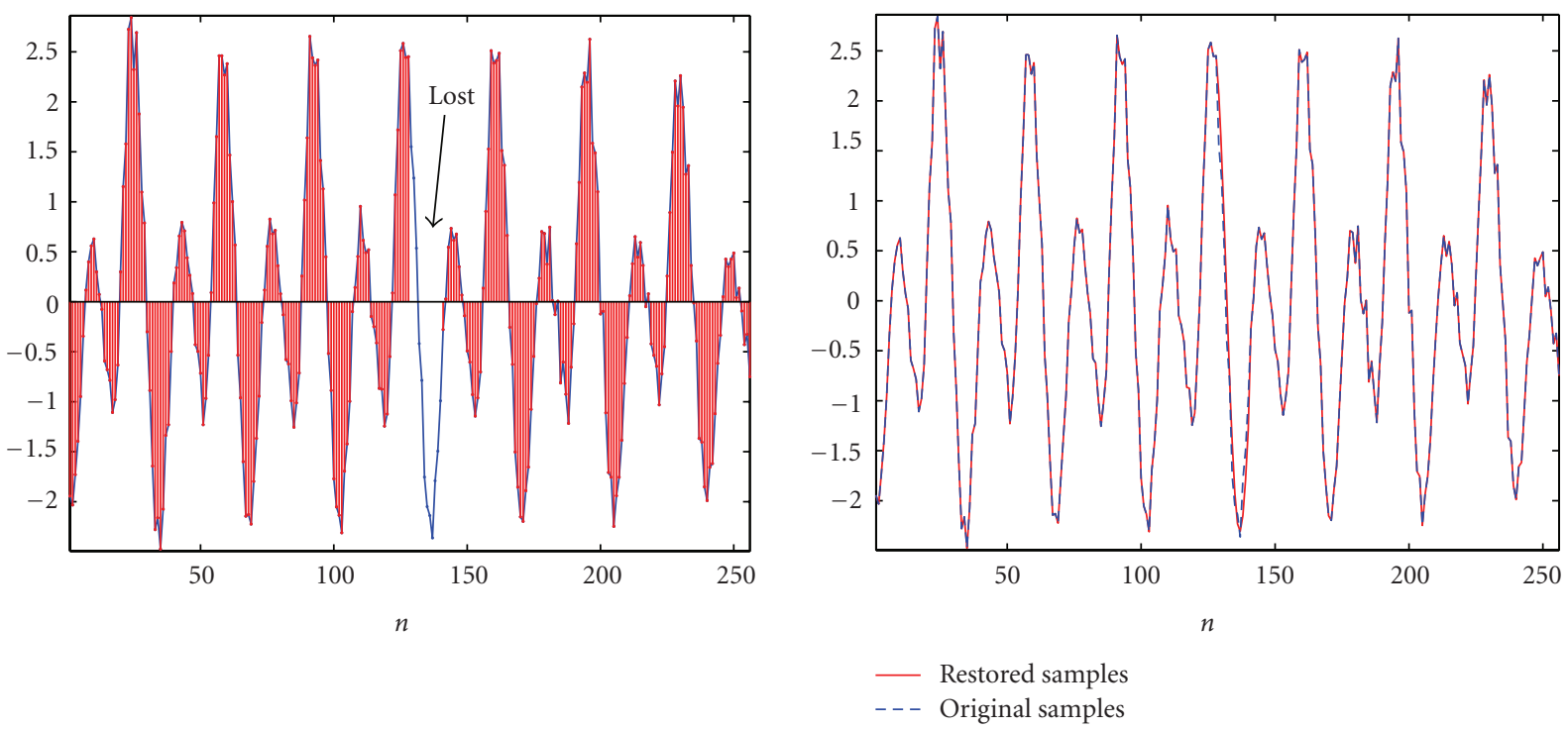

(a)
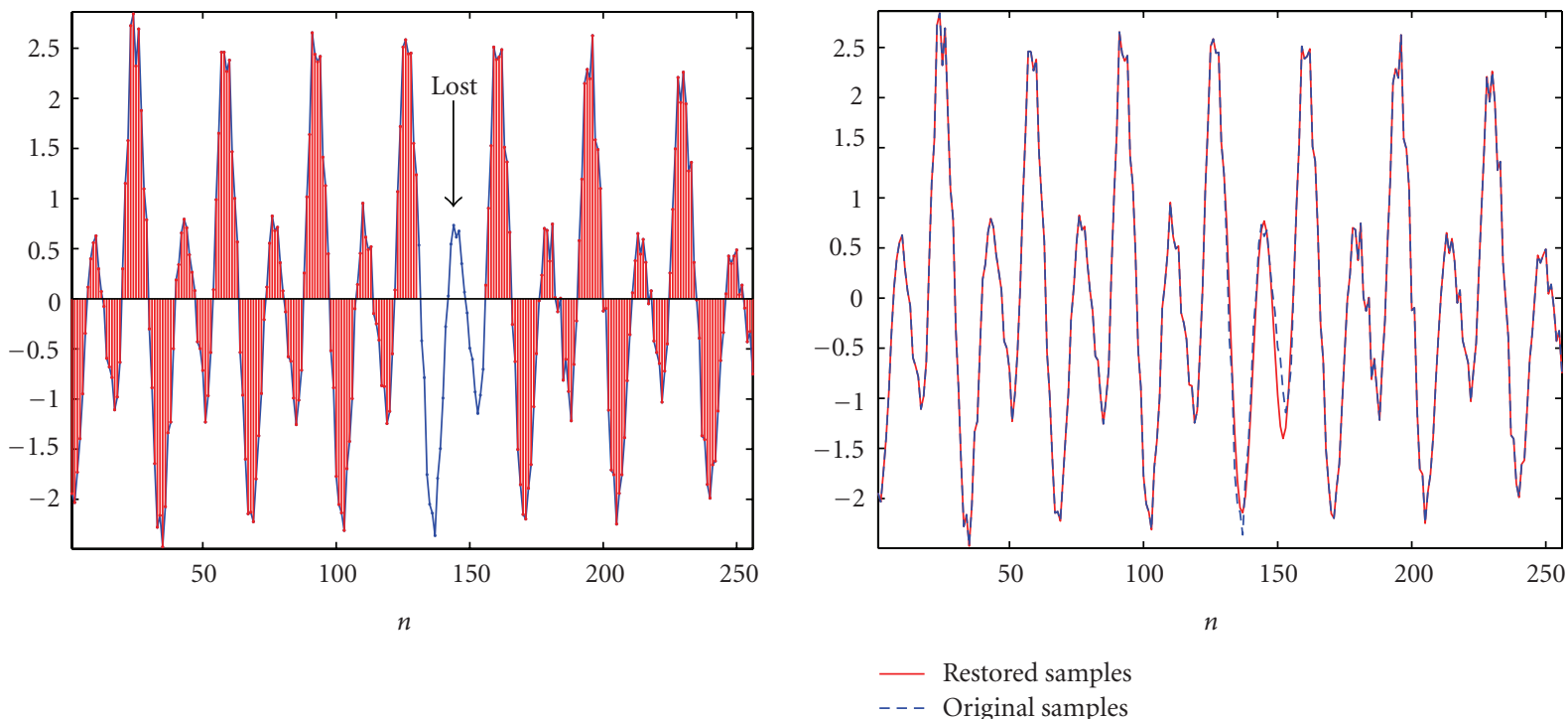

(b)

FIGURE 9: Restored results for continuous lost samples. (a) Restored speech signal from 12 missing data after 10 iterations and (b) restored speech signal from 25 missing data after 70 iterations.

accuracy obtained from baseband DPSS, and then restored by small number of projection iteration with the same MAE performance compared to baseband DPSS-based DET. The DET-based POCS algorithm is shown to provide fast and accurate technique for recovering the band-limited samples from the irregularly spaced subsamples. Although there are remaining issues that need further study, for instance, the upper error bound by the number and distribution of missing samples, the proposed method shows very promising results, that is, capable of signal recovery from randomly spaced subsamples and continuous lost samples.

\section{References}

[1] L. M. Bregman, "Finding the common point of convex sets by the method of successive projections," Doklady Akademii Nauk SSSR, vol. 162, no. 3, pp. 487-490, 1965.

[2] L. G. Gubin, B. T. Polyak, and E. V. Raik, "The method of projections for finding the common point of convex sets," USSR Computational Mathematics and Mathematical Physics, vol. 7, no. 6, pp. 1-24, 1967.

[3] S. Yeh and H. Stark, "Iterative and one-step reconstruction from nonuniform samples by convex projections," Journal of the Optical Society of America, vol. 7, no. 3, pp. 491-499, 1990. 
[4] R. Stasiński and J. Konrad, "POCS-based image reconstruction from irregularly-spaced samples," in Proceedings of the IEEE International Conference on Image Processing (ICIP '00), vol. 2, pp. 315-318, September 2000.

[5] J. Park, D.-C. Park, R. J. Marks II, and M. A. El-Sharkawi, "Block loss recovery in DCT image encoding using POCS," in Proceedings of the IEEE International Symposium on Circuits and Systems, vol. 5, pp. 245-248, September 2002.

[6] H. Huang and A. Makur, "A new iterative reconstruction scheme for signal reconstruction," in Proceedings of the IEEE Asia Pacific Conference on Circuits and Systems (APCCAS '08), pp. 336-339, December 2008.

[7] T. Ogawa and M. Haseyama, "Adaptive reconstruction method of missing texture based on projection onto convex sets," in Proceedings of the IEEE International Conference on Acoustics, Speech and Signal Processing (ICASSP '07), vol. 1, pp. 697-700, April 2007.

[8] J. Chen, L. Zhang, J. Luo, and Y. Zhu, "MRI reconstruction from 2D partial k-space using POCS algorithm," in Proceedings of the 3rd International Conference on Bioinformatics and Biomedical Engineering (ICBBE '09), pp. 1-4, June 2009.

[9] H. G. Feichtinger, W. Kozek, and T. Strohmer, "Reconstruction of signals from irregular samples of its short-time Fourier transform," in Wavelet Applications in Signal and Image Processing III, Proceedings of SPIE, pp. 140-150, San Diego, Calif, USA, July 1995.

[10] H. E. Guven, H. M. Ozaktas, A. E. Cetin, and B. Barshan, "Signal recovery from partial fractional Fourier domain information and its applications," IET Signal Processing, vol. 2, no. 1, pp. 15-25, 2008.

[11] A. Serbes and L. Durak, "Optimum signal and image recovery by the method of alternating projections in fractional Fourier domains," Communications in Nonlinear Science and Numerical Simulation, vol. 15, no. 3, pp. 675-689, 2010.

[12] T. Strohmer, "Numerical analysis of the non-uniform sampling problem," Journal of Computational and Applied Mathematics, vol. 122, no. 1, pp. 297-316, 2000.

[13] G. Walters and X. Shen, "Sampling with prolate spheroidal wave functions," Sampling Theory in Signal and Image Processing, vol. 2, pp. 25-52, 2003.

[14] S. Senay, L. F. Chaparro, and L. Durak, "Reconstruction of nonuniformly sampled time-limited signals using prolate spheroidal wave functions," Signal Processing, vol. 89, no. 12, pp. 2585-2595, 2009.

[15] D. Slepian and H. O. Pollak, "Prolate spheroidal wave functions, Fourier analysis and uncertainty," Bell System Technical Journal, vol. 40, pp. 43-64, 1961.

[16] D. Slepian, "Prolate spheroidal wave functions, Fourier analysis and uncertainty. V: the discrete case," Bell System Technical Journal, vol. 57, no. 5, pp. 1371-1430, 1978.

[17] J.-J. Ding and S.-C. Pei, "Reducing sampling error by prolate spheroidal wave functions and fractional Fourier transform," in Proceedings of the IEEE International Conference on Acoustics, Speech, and Signal Processing (ICASSP '05), vol. 4, pp. 217220, March 2005.

[18] M. B. Priestley, Non-Linear and Non-Stationary Time Series Analysis, Academic Press, London, UK, 1988.

[19] R. Suleesathira, L. F. Chaparro, and A. Akan, "Discrete evolutionary transform for time-frequency signal analysis," Journal of the Franklin Institute, vol. 337, no. 4, pp. 347-364, 2000.

[20] M. Unser, “Sampling-50 years after Shannon," Proceedings of the IEEE, vol. 88 , no. 4, pp. 569-587, 2000.
[21] E. Sejdić, M. Luccini, S. Primak, K. Baddour, and T. Willink, "Channel estimation using DPSS based frames," in Proceedings of the IEEE International Conference on Acoustics, Speech and Signal Processing (ICASSP '08), pp. 2849-2852, March 2008.

[22] S. Şenay, L. F. Chaparro, and A. Akan, "Chirp channel estimation and OFDM transmission using discrete prolate spheroidal sequences," in Proceedings of the 5th IASTED International Conference on Signal Processing, Pattern Recognition, and Applications (SPPRA '08), vol. 64, pp. 281-286, February 2008.

[23] P. J. S. G. Ferreira, "The stability of a procedure for the recovery of lost samples in band-limited signals," Signal Processing, vol. 40, no. 2-3, pp. 195-205, 1994.

[24] P. Oliveira, "Interpolation of signals with missing data using PCA," in Proceedings of the IEEE International Conference on Acoustics, Speech and Signal Processing (ICASSP '06), vol. 3, pp. 828-831, May 2006. 